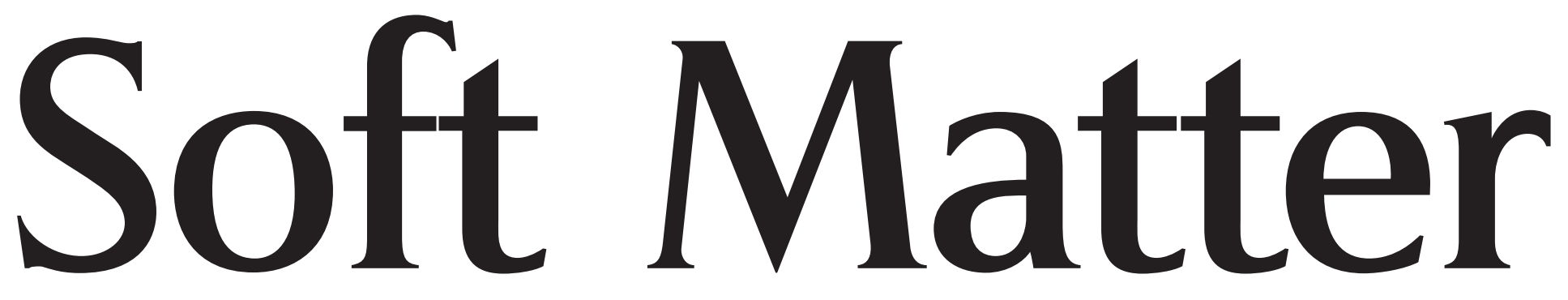




\title{
Temperature dependent stiffness and visco-elastic behaviour of lipid coated microbubbles using atomic force microscopy
}

\author{
Colin A. Grant, $\uparrow$ Jonathan E. McKendry and Stephen D. Evans* \\ Received 18th August 2011, Accepted 30th September 2011 \\ DOI: $10.1039 / \mathrm{c} 1 \mathrm{sm} 06578 \mathrm{e}$
}

\begin{abstract}
The compression stiffness of a phospholipid microbubble was determined using force-spectroscopy as a function of temperature. The stiffness was found to decrease by approximately a factor of three from $\sim 0.08 \mathrm{~N} \mathrm{~m}^{-1}$, at $10{ }^{\circ} \mathrm{C}$, down to $\sim 0.03 \mathrm{~N} \mathrm{~m}^{-1}$ at $37^{\circ} \mathrm{C}$. This temperature dependence indicates that the surface tension of lipid coating is the dominant contribution to the microbubble stiffness. The timedependent material properties, e.g. creep, increased non-linearly with temperature, showing a factor of two increase in creep-displacement, from $\sim 24 \mathrm{~nm}$, at $10^{\circ} \mathrm{C}$, to $50 \mathrm{~nm}$, at $37^{\circ} \mathrm{C}$. The standard linear solid model was used to extract the visco-elastic parameters and their determination at different temperatures allowed the first determination of the activation energy for creep, for a microbubble, to be determined.
\end{abstract}

\section{Introduction}

Micron-sized, gas microbubbles encapsulated within a thin shell of lipid ${ }^{1-6}$ or other surfactant based material ${ }^{7-11}$ are generating increasing interest as ultrasound contrast agents (UCA's) ${ }^{12-14}$ and potential drug delivery vehicles. ${ }^{12-18}$ It is well documented that such microbubbles dissolve almost instantaneously in the absence of their surfactant coating ${ }^{3}$ and that the coating not only affects the resistance to gas permeation, and hence microbubble lifetime, but also their mechanical properties. ${ }^{19-22}$

The use of lipid based coatings is widespread not only because of their intrinsic biocompatibility but also because lipid based engineering is relatively advanced, permitting the attachment of PEG chains to reduce non-specific interactions and increase biocompatibility and functional groups, such as antibodies or peptides, for targeting. ${ }^{23}$ Furthermore the wide range of lipids available has allowed fundamental studies on the role of the coatings in controlling the microbubble stiffness, viscosity and strain behaviour. ${ }^{24}$

Recently, we have shown that the compression stiffness of phospholipid microbubbles is significantly increased, by a factor of 30, via the adsorption of a streptavidin layer at bilayer/water interface. ${ }^{25}$ Other research on microbubbles has determined the stiffness and elastic modulus of 'thick-shelled' and 'hollow thinshelled' polymeric microbubbles. ${ }^{9-11}$

To date, however, all such force spectroscopy (FS) studies have been undertaken at room temperature and therefore

Molecular Nanoscale Physics Group, School of Physics and Astronomy, University of Leeds, Leeds, LS2 9JT, UK. E-mail: s.d.evans@leeds.ac. uk; Tel: +44 (0)1133433852

$\uparrow$ Present address: Advanced Materials Engineering RKT Centre, School of Engineering, Design and Technology, University of Bradford, Bradford, BD7 1DP, UK. unlikely to represent the behaviour expected in vivo. Further, whilst the mechanical properties of such microbubbles are commonly characterised as being isotropic, elastic and timeindependent, they may in fact be better understood as viscoelastic materials exhibiting time (or rate) dependent properties. Traditionally, there are two ways of testing visco-elastic timedependent behaviour via nanoindentation: $i$ ) monitoring the displacement under a constant stress (creep) or, ii) monitoring the load under a constant strain (relaxation). Creep and relaxation testing has been successfully carried out, at the micro-level, on cartilage and bone ${ }^{26}$ and recently on living cells. ${ }^{27}$ It is also worth noting that the visco-elastic properties of materials are generally known to be sensitive to temperature. ${ }^{28,29}$

In this paper we present the first report on the use forcespectroscopy to determine the mechanical properties of lipidcoated microbubble as a function of temperature and loading rate. We also present the first studies on the determination of their visco-elastic properties, by analysing the creep response, as a function of temperature. The methodology and results obtained provide a starting point for the future design microbubble shells with desirable visco-elastic responses.

\section{Results and discussion}

Using the AFM optical microscope, a suitable microbubble is located (Fig. 1a). The width of the cantilever $(50 \mu \mathrm{m})$ is used to provide an approximate measure the diameter of the microbubble. A generalised schematic showing the cantilever and bubble arrangement is shown in Fig. $1 \mathrm{~b}$.

Fig. 2 shows a typical force-spectroscopy plot of a creep experiment on a microbubble. The three separate stages, shown in Fig. 2a, represent loading, hold and unloading. Initially, the microbubble is subject to an increasing load (loading curve) to 


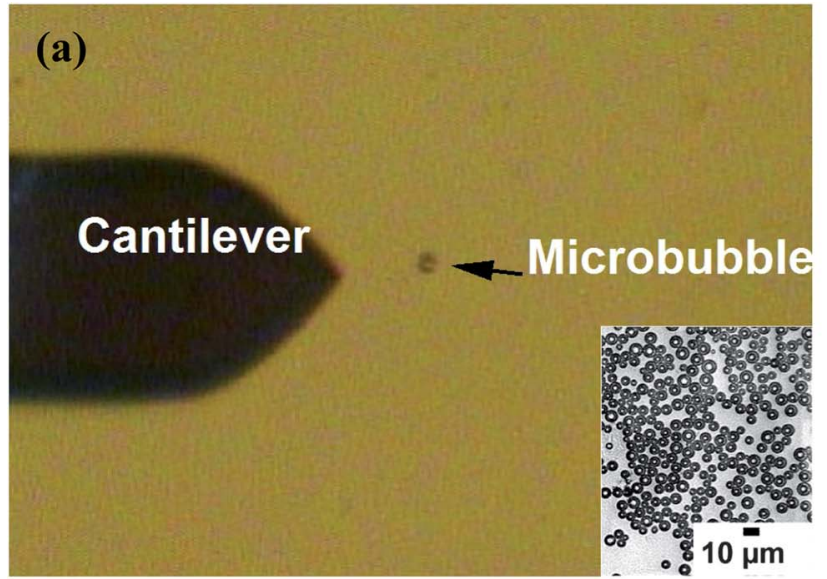

(b)

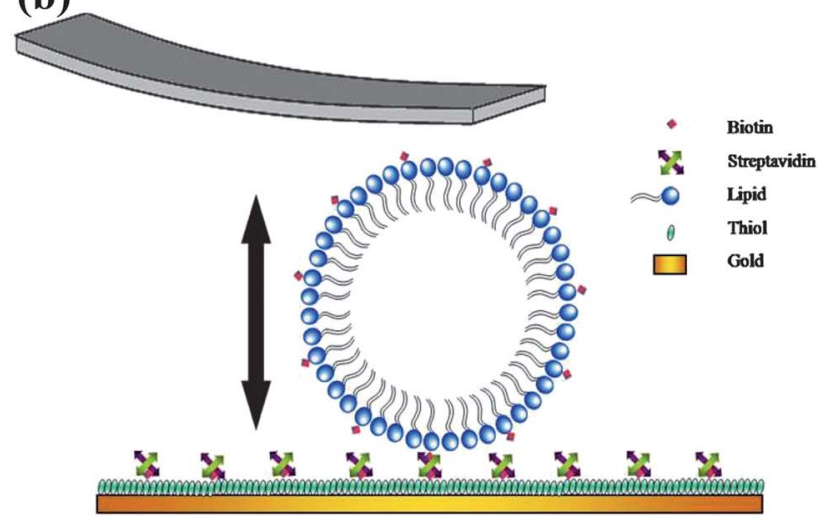

Fig. 1 (a) AFM optical image of the cantilever (width $=50 \mu \mathrm{m}$ ) with a suitable isolated lipid microbubble (inset) production of microbubbles from micro-fluidic device (b) schematic diagram highlighting the cantilever/microbubble system.

a set value at which the force is maintained at a constant value for a fixed period of time (hold), Fig. 2b (lower). During this "hold" period the displacement versus time is recorded, Fig. 2b (upper) and subsequently the load is removed (unloading curve). The process was repeated at least 50 times to check repeatability and to allow mean values to be obtained. It is worth noting that the curves were highly reproducible, indicating no loss of gas during these loading/unloading cycles. Overall, the force curves (load/ hold/unload) were smooth and exhibited no instabilities as observed by Glynos et al. ${ }^{9}$ As expected for a visco-elastic solid, the force curves show hysteresis (area bound by the load/hold/ unload and the line $\mathrm{F}=0$ ), due to heat loss or dissipation during the indentation. However, our indentations remain in the elastic region as demonstrated by the repeated force curves (1st and 50th) overlaying each other. Furthermore, the adhesive break-off point, from the unloading curve, occurs approximately at the zero-contact point (Fig. 2b); highlighting no plastic deformation. The observed adhesion on unloading might be due to increased bubble-substrate or bubble-tip interactions; however this is both reversible and small. The displacement was calculated as the difference between the change of z-piezo displacement and the change in tip deflection, i.e. indentation depth. However, as the bubble system can be compressed at both its poles, we use the term 'displacement' for clarity.

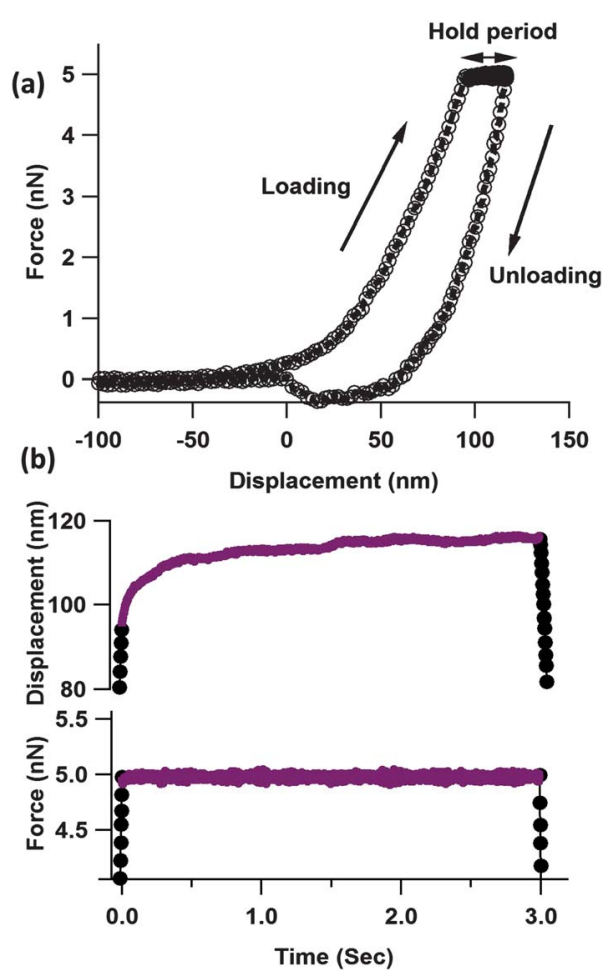

Fig. 2 (a) Typical force versus displacement cycle on DPPC: PE-biotin $(9: 1)$ coated microbubble, showing the loading, hold and unloading sections, (b) displacement (upper) force (lower) traces versus time - during the hold period (solid line).

The force-displacement curves were non-linear in the low applied force regime, and hence the gradient, which represents the microbubble stiffness, was obtained by fitting a straight line to the upper portion of the curves as shown by the red lines in Fig. 3a. The force displacement curve displays a non-linear behaviour at low applied force $(<2 \mathrm{nN})$, due to a combination of increasing contact area between the micro-bubble and the tip and also due to deformation at the micro-bubble/substrate interface. As a result it is unlikely that at low applied force $(<2 \mathrm{nN})$ one is measuring purely the mechanical properties of the microbubble. Consequently, the stiffness values were obtained by fitting a straight line (shown as solid line in fig. 3a) to the portion of the curves for which the applied force was greater than $3 \mathrm{nN}$. Fig. $3 \mathrm{a}$ shows the force-displacement curves obtained from a single microbubble at 4 different temperatures between 10 and $37^{\circ} \mathrm{C}$. It is evident from decrease in slope that the compression stiffness decreases significantly with increasing temperature. For the same applied load, $5 \mathrm{nN}$, the displacement observed at $10{ }^{\circ} \mathrm{C}$ only reaches $\sim 80 \mathrm{~nm}$ whereas for measurements made at $37^{\circ} \mathrm{C}$, the displacement depth reaches over $200 \mathrm{~nm}$. Whilst this is a significant increase it is still less than $5 \%$ of the microbubble diameter, thus allowing the displacement to be treated as in a low strain regime.

The stiffness measured in such force spectroscopy measurements arises due to a combination of the bulk modulus of the gas and stiffness due to the lipid shell. Increasing the temperature could either lead to an increase in the gas pressure, which would concomitantly lead to an increase in the stiffness due to the gas core (the opposite to that observed) or to an increase in bubble 

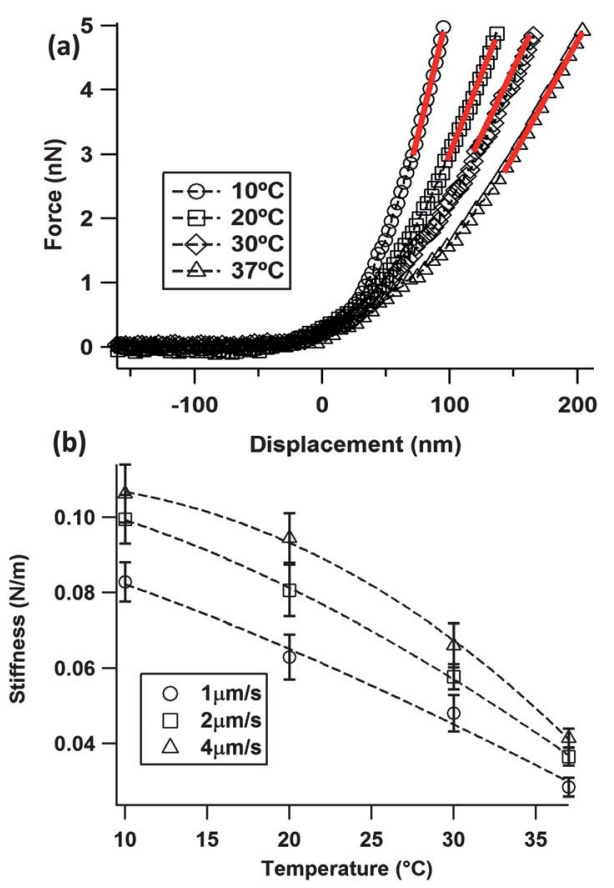

Fig. 3 (a) Force $v s$. displacement plots made on a single MB at different temperatures between 10 and $37^{\circ} \mathrm{C}$, at a constant velocity $\left(1 \mu \mathrm{m} \mathrm{s}^{-1}\right)$. The solid lines represent straight line fits used to obtain stiffness values (b) Compression stiffness vs. temperature, obtained at three different tip velocities - each point represents the mean \pm standard deviation obtained from 50 loading cycles.

volume which would lead to a softer bubble..$^{25}$ However, for the temperature range considered here, we would only estimate $\sim 9 \%$ increase in volume (corresponding to a $\sim 3 \%$ increase in radius) and from our previous work expect this to correspond to only a change in stiffness of $\sim 1 \mathrm{mN} \mathrm{m}^{-1}$. In contrast, increasing the temperature would also be expected to reduce the lateral interactions between lipids, thereby reducing the surface tension and lead to a reduction in the stiffness values determined from the force spectroscopy. Experimentally, it is difficult to determine optically whether such bubbles change size, as our spatial resolution is only $\sim 0.4 \mu \mathrm{m}$. However, we would expect to be sensitive to changes in bubble size in the force-displacement curves (i.e. the location of the point of zero contact) where we did not observe a notable expansion. Thus, the significant decrease in stiffness observed with increasing temperature indicates that the properties of the lipid shell dominate the force-spectroscopy measurements (Fig. 3b). Measurement of the nano-mechanical properties of supported bilayers have also shown a reduction in the lateral interaction between lipid molecules, with increasing temperature, leading to a reduction in the breakthrough force required to penetrate the bilayer. ${ }^{30}$ Furthermore, course-grain lipid modelling showed that acyl chain tails became increasingly disordered with increasing temperature. ${ }^{31}$

Fig. $3 \mathrm{~b}$ shows that the stiffness varies inversely with temperature and also that there was a weak dependence on loading speed. We note that the stiffness plots converge towards a value of $\sim 0.03 \pm 0.002 \mathrm{~N} \mathrm{~m}^{-1}\left(1 \mu \mathrm{m} \mathrm{s}^{-1}\right)$, at $37^{\circ} \mathrm{C}$, nearly a factor of three lower than obtained for $10^{\circ} \mathrm{C}$. Whilst values of stiffness as determined above provides a useful parameter for understanding and comparing the mechanical the response of microbubbles to external forces, under varying loading rates and temperatures, it does not provide a full characterisation of its visco-elastic, timedependent, behaviour.

In order to assess the visco-elastic contribution in the force plot, we have examined the creep response. Plots of the displacement versus time obtained during the "hold" period are shown, in Fig. 4a, for each temperature. The displacement creep data is a measure of the maximum change in deformation whilst a constant load is applied for a period of $3 \mathrm{~s}$. This was then repeated at 3 different loading velocities and at 4 different temperatures (Fig.4b).

The visco-elastic creep experienced by a lipid microbubble is non-linearly dependent upon temperature. At the lowest temperature $\left(10{ }^{\circ} \mathrm{C}\right)$ the measured creep displacement is small, $\sim 20 \mathrm{~nm}$ and is relatively insensitive to the loading speed. Whereas, at $37^{\circ} \mathrm{C}$ the measured displacement creep is $\sim 40-50$ $\mathrm{nm}$ and shows a slightly stronger dependence on loading rate.

It is possible to fit the creep displacement $v s$. time curves of Fig. 4(a) to a simple mechanical model, the so-called standard linear solid model combines a purely elastic element $\left(\mathrm{k}_{1}\right)$ and a visco-elastic Maxwell element $\left(\mathrm{k}_{0}, \eta_{0}\right)$ arranged in parallel, Fig. 5(a). Simpler models to analyse linear visco-elastic behaviour, such as the Maxwell or Kelvin-Voigt, do not adequately model creep or stress relaxation, whereas the standard linear solid model is the most basic model that describes both phenomena. The model may have no molecular basis but represents a simple combination to examine the visco-elastic behaviour of the microbubble. The fit to eqn (1) in Fig. 5b represents the behaviour of the standard linear solid following
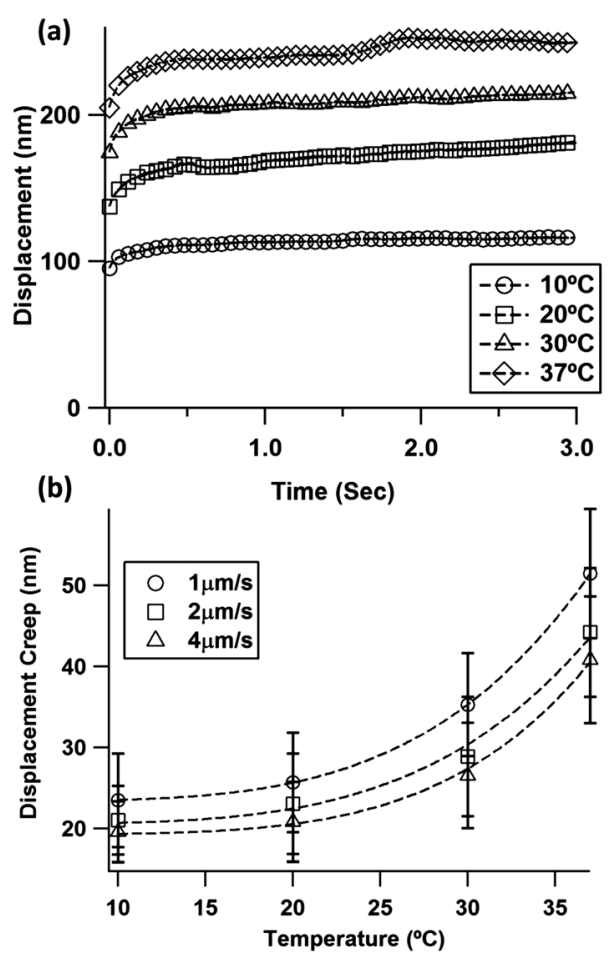

Fig. 4 (a) Typical creep displacement curves obtained for a microbubble held at different temperatures between 10 and $37{ }^{\circ} \mathrm{C}$. (b) Mean displacement creep vs. temperature, at three different loading velocities. 
(a)

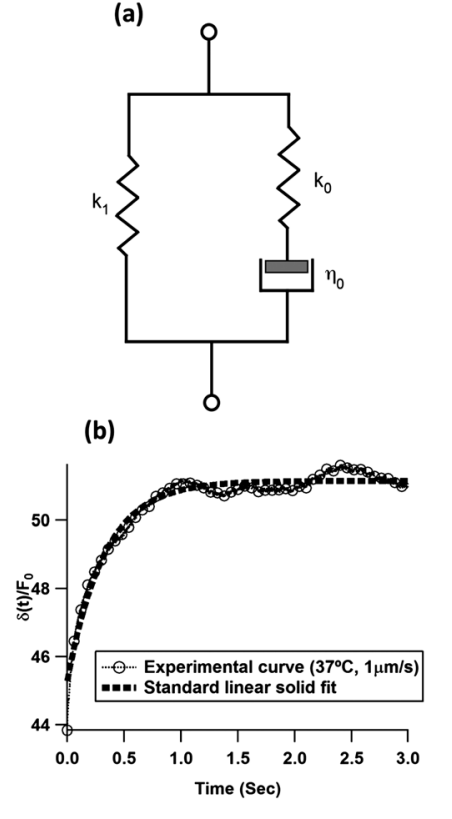

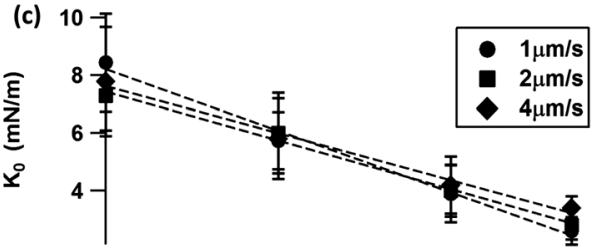
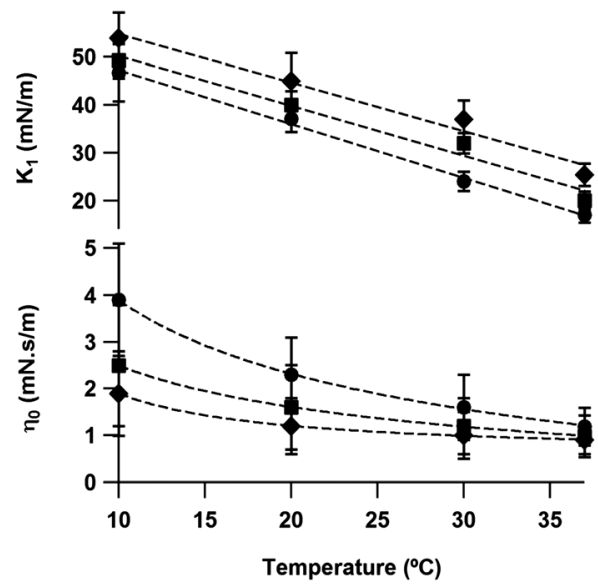

Fig. 5 (a) Standard linear model comprising of an elastic element, $k_{0}$, in parallel with a visco-elastic Maxwell element, $k_{1}$, $\eta_{0}$. (b) 'Normalised' creep displacement curve taken at $37 \mathrm{C}, 1 \mu \mathrm{m} \mathrm{s}^{-1}$ with fit to the standard linear solid model, (c) Results of model fitting results of $\mathrm{k}_{0}$, $\mathrm{k}_{1}$ and $\eta_{0}$ at varying temperature and loading velocity.

a unit step increase in stress, where the creep has been normalised with the applied load.

The results of the fitting show a linear decrease of stiffness, of the elastic components, $\mathrm{k}_{0}$ and $\mathrm{k}_{1}$ with increasing temperature. The damping coefficient, as calculated using eqn (2), $\eta_{0}$ also shows a non-linear decrease with increasing temperature and is influenced more by loading velocity (Fig. 5(c)). Results from the fitting to the standard linear solid model are tabulated in Table 1.

The time dependent creep behaviour, as modelled by springs/ dashpots, has been carried out on polymers, ${ }^{32}$ bone, ${ }^{33,34}$ and more recently on single bacterial cells $\mathrm{s}^{35}$ and bacterial biofilms, ${ }^{36}$

A method of extracting the activation energy of creep $(Q)$ by examination of the strain rate behaviour over a range of temperatures has been commonly used for metals/ceramics, using eqn (3) and (4) ${ }^{37}$ However, to the best of our knowledge, this has

Table 1 Curve fitting results of microbubble visco-elastic behaviour with varying temperature and loading rate

\begin{tabular}{lllll}
\hline & $\begin{array}{l}\text { Tip velocity } \\
T /{ }^{\circ} \mathrm{C}\end{array}$ & & & \\
\hline \multirow{2}{*}{10} & 1 & $\mathrm{~K}_{0}\left(\mathrm{mN} \mathrm{m}^{-1}\right)$ & $\mathrm{K}_{1}\left(\mathrm{mN} \mathrm{m}^{-1}\right)$ & $\eta_{0}\left(\mathrm{mN} \mathrm{s} \mathrm{m}^{-1}\right)$ \\
& 2 & $7.45 \pm 1.7$ & $46.6 \pm 6.1$ & $3.9 \pm 1.2$ \\
& 4 & $7.8 \pm 1.2$ & $49.3 \pm 3.7$ & $2.5 \pm 1.3$ \\
20 & 1 & $5.7 \pm 1.1$ & $53.9 \pm 5.3$ & $1.9 \pm 0.9$ \\
& 2 & $6.0 \pm 1.4$ & $40.1 \pm 2.8$ & $2.3 \pm 0.8$ \\
30 & 4 & $5.8 \pm 1.4$ & $45.3 \pm 5.8$ & $1.6 \pm 0.9$ \\
& 1 & $3.9 \pm 1.0$ & $24.1 \pm 2.3$ & $1.2 \pm 0.6$ \\
& 2 & $4.1 \pm 0.9$ & $32.0 \pm 2.1$ & $1.2 \pm 0.6$ \\
37 & 4 & $4.2 \pm 1.1$ & $37.3 \pm 3.9$ & $1.0 \pm 0.5$ \\
& 1 & $2.6 \pm 0.5$ & $17.2 \pm 1.6$ & $1.2 \pm 0.4$ \\
& 2 & $2.8 \pm 0.5$ & $20.3 \pm 1.9$ & $0.98 \pm 0.45$ \\
& 4 & $3.4 \pm 0.4$ & $25.4 \pm 2.3$ & $0.9 \pm 0.3$ \\
\hline
\end{tabular}

yet been applied to microbubbles, vesicles or cells. The strain rate was calculated using eqn (3), where $\Delta \delta / \delta t$ is the gradient at a constant level of displacement creep depth as shown in Fig. 6 (a). For these experiments, we set the level at $h=15 \mathrm{~nm}$, however,
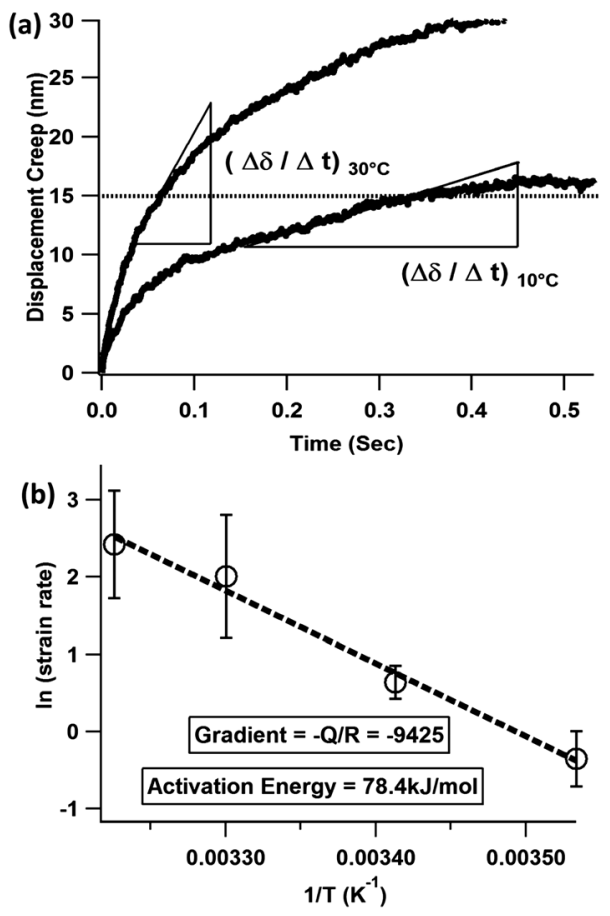

Fig. 6 (a) Strain rate dependence varying as a function of temperature, (b) plot of $\ln$ (strain rate) against $1 / T$, where the activation energy can be deduced from the gradient of such a plot. 
some of the curves taken at $10{ }^{\circ} \mathrm{C}$ did not have this amount of creep and were not included in the analysis.

Fig. 6(b) shows the plot of $\ln$ (strain rate) against $1 / \mathrm{T}$, where the gradient is equal to $-Q / R$. The activation energy for creep, of a microbubble, was determined to be $78 \mathrm{~kJ} \mathrm{~mol}^{-1}$. Such an approach been applied to more biologically relevant samples, collagen fibrils, where $Q$ was found to be $50 \mathrm{~kJ} \mathrm{~mol}^{-1} \cdot{ }^{38}$ Due to the limited determination of the activation energy of creep, via AFM measurements, it is difficult to put our value for $Q$ in to context. Though, it is hoped it will provide starting point for future refinement and allow comparison for bubbles with different shell properties.

\section{Experimental}

\section{Microbubble preparation}

Phospholipid coated microbubbles were prepared using the method described by McKendry et al. ${ }^{25}$ in which a flow focussed microfluidic chip was used to create gas $\left(\mathrm{C}_{3} \mathrm{~F}_{8}\right)$ filled microbubbles. A gas pressure of $76 \mathrm{kPa}$ and liquid flow rate of $50 \mu \mathrm{L}$ $\mathrm{s}^{-1}$ were optimal for producing microbubbles of 2-8 $\mu \mathrm{m}$ diameter. The phospholipid coat composition consisted of a 90 : 10 mixture of 1,3-Dipalmitoyl-sn-glycero-3-phosphocholine (DPPC) to 1-oleoyl-2-(12-biotinyl(aminolauroyl))-sn- $g l y c$ ero-3-phosphoethanolamine (PE-biotin).

The microbubbles to be studied were immobilized on a biotinylated self-assembled monolayer (SAM), on a gold-coated glass cover slip. The SAMs were formed by incubation of a freshly prepared gold surface for $12 \mathrm{~h}$, in a $15 \mathrm{mM}$ methanolic solution containing a mixture $(90: 10 \mathrm{~mol}$. \%) of 6-mercaptohexanol (Sigma- Aldrich, Poole, UK) and a biotinylated thiol (Assemblon, WA USA). For the attachment of the lipid-coated microbubbles the SAM was first incubated for $2 \mathrm{~h}$ in a $0.1 \mathrm{mg} \mathrm{mL}^{-1}$ solution of streptavidin. Following streptavidin attachment the cover slip was placed in contact with the microbubble solution in an inverted manner for a period of $1 \mathrm{~h}$. This facilitated the attachment of floating microbubbles via a biotin-streptavidin bridge.

\section{AFM sample preparation and calibration}

The sample was then rinsed with excess MilliQ water to remove unattached microbubbles from the SAM surface and placed onto the AFM fluid cell (MFP3D Asylum Research, Santa Barbara, USA) and attached to a Peltier heater/cooling device. The microbubbles were maintained within $2 \mathrm{~mL}$ of buffer solution (1 vol\% glycerine (Sigma-Aldrich, St. Louis, USA) 99 vol\% MilliQ water solution containing $4 \mathrm{mg} \mathrm{ml}^{-1} \mathrm{NaCl}$.). The heater was warmed to the appropriate temperature $\left(10,20,30\right.$ and $\left.37^{\circ} \mathrm{C}\right)$ and allowed to equilibrate for an hour, before calibrating the spring constant of the cantilever, using the thermal tuning method. ${ }^{39}$ The laser sensitivity was measured by pressing the cantilever on to the hard gold substrate once the system had reached its thermal equilibrium. For the range of experimental temperatures, the measured cantilever spring constant $\left(0.17 \mathrm{~N} \mathrm{~m}^{-1}\right)$ varied by less than $4 \%$.

\section{AFM mechanical testing}

A suitable individual microbubble was then located using the AFM optics. A digital image was captured in order to measure the microbubble diameter (Fig. 1); the AFM tipless cantilever (Nanosensors, UK), with known width $(50 \mu \mathrm{m})$ was used as a calibration for determination of the microbubble diameter (4.5 $\mu \mathrm{m})$. The cantilever was then moved directly above the microbubble and slowly brought in to soft contact. Fifty force curves were recorded in closed $\mathrm{z}$-loop mode with a range of tip velocities $\left(1,2\right.$ and $\left.4 \mu \mathrm{m} \mathrm{s}^{-1}\right)$ and for a series of temperatures between 10 and $37^{\circ} \mathrm{C}$ ).

\section{Stiffness analysis}

Stiffness values were determined by measuring the gradient of the force-displacement plot (Fig. 3a) for each tip velocity, and at each temperature. The gradient of the recorded forcedisplacement plots was used to determine the contact stiffness of the cantilever/microbubble system $\left(k_{S Y S}\right)$. The compression of thin shelled spheres modeled as a two springs in series ${ }^{40}$ with the stiffness of the microbubble $\left(k_{M B}\right)$ can be given by $1 /$ $k_{M B}=1 / k_{S Y S}-1 / k_{c}$, where $k_{c}$ is the spring constant of the cantilever.

The maximum load for the force curves was maintained at 5 $\mathrm{nN}$; this was then followed by a $3 \mathrm{~s}$ hold in order to maintain a constant force on the microbubble to assess the creep behaviour (Fig. 2b). The feedback for the hold section was set to monitor the cantilever deflection, which is directly proportional to the applied load. The amount of creep, continuation of deformation of material under constant stress, was recorded during this hold section.

\section{Creep analysis}

A standard linear solid model comprising of a Maxwell element (spring/dashpot in series) in parallel with a dashpot (Fig. 5a) is applied to the creep vs. time curves made on the micro-bubbles at different tip velocities and temperatures. It can be shown that the time dependent $(\delta(t))$ creep behaviour of the standard linear solid model, following a step change in applied force $\left(F_{0}\right)$, can be described by eqn (1).

$$
\frac{\delta(t)}{F_{\mathrm{o}}}=\frac{1}{k_{o}}\left(1-\frac{k_{o}}{k_{o}-k_{1}} e^{-t / \tau}\right)
$$

Where $k_{0}, k_{1}$ are the spring constants of the two spring elements

$$
\eta_{o}=\tau\left(\frac{k_{o} k_{1}}{k_{o}+k_{1}}\right)
$$

Further, the relaxation time $(\tau)$ of the visco-elastic material is used to calculate $\eta_{O}$, which is the damping coefficient of the dashpot using eqn (2).

The maximum amount of creep is extracted from the displacement creep vs. time curves over the $3 \mathrm{~s}$ hold for each of the 3 different loading rates. The strain rate was calculated using eqn (3) and activation energy $Q$ was calculated using the standard creep eqn (4). The strain rate was taken at a constant creep displacement depth $(\delta)$, where the gradient $(\Delta \delta / \Delta t)$ was recorded.

$$
\begin{gathered}
\dot{\varepsilon}=\frac{1}{\delta} \frac{\Delta \delta}{\Delta t} \\
\dot{\varepsilon}=C \sigma^{n} \exp (-Q / R T)
\end{gathered}
$$


Where $C, \sigma$ and $n$ are material constants, $R$ the universal gas constant, and $T$ the temperature $(\mathrm{K})$.

\section{Conclusions}

The stiffness and visco-elastic properties of microbubbles are important quantities, which will inform their use as agents for therapeutic delivery. In particular, control over the composition and architecture of microbubble coatings is expected to play a significant role in determining their visco-elastic response. The force spectroscopy studies presented here have shown that the stiffness of lipid-coated microbubbles decreased by nearly a factor of 3 as the temperature was increased from 10 to $37^{\circ} \mathrm{C}$.

Further, these systems also displayed temperature dependent visco-elastic creep behaviour, on a single microbubble, found to nearly double on increasing the temperature to $37^{\circ} \mathrm{C}$. Whilst the interpretation in terms of a molecular model is not yet available, an activation energy for creep was determined to be $78 \mathrm{~kJ} \mathrm{~mol}^{-1}$ compared to that of $50 \mathrm{~kJ} \mathrm{~mol}^{-1}$ for collagen fibrils.

Our results indicate the need to perform combined stressrelaxation and creep studies of lipid-coated microbubbles, with defined architectures and composition, to permit the extraction of physically meaningful parameters from the visco-elastic modelling.

\section{Acknowledgements}

This research was sponsored by the EPSRC (EP/I000623). We gratefully acknowledge the support Leeds Microbubble Consortium $\$$ Also, we would like to acknowledge Dr Lars Jeuken (University of Leeds, School of Physics) for creating the graphical abstract.

\section{References}

1 T. Faez, D. Goertz and N. De Jong, Ultrasound Med. Biol., 2011, 37 (2), 338-342.

2 U. Farook, E. Stride and M. J. Edirisinghe, Eur. Biophys. J., 2009, 38 (5), 713-718.

3 P. R. K. Ferrara and M. Borden, Annu. Rev. Biomed. Eng., 2007, 9, 415-447.

4 K. Hettiarachchi, E. Talu, M. L. Longo, P. A. Dayton and A. P. Lee, Lab Chip, 2007, 7(4), 463-468.

5 V. Sboros, Adv. Drug Delivery Rev., 2008, 60(10), 1117-1136.

6 E. Talu, M. M. Lozano, R. L. Powell, P. A. Dayton and M. L. Longo, Langmuir, 2006, 22(23), 9487-9490.

7 N. de Jong, R. Cornet and C. T. Lancee, Ultrasonics, 1994, 32(6), 455459.

8 N. de Jong and L. Hoff, Ultrasonics, 1993, 31(3), 175-181.
9 E. Glynos, V. Koutsos, W. N. McDicken, C. M. Moran, S. D. Pye, J. A. Ross and V. Sboros, Langmuir, 2009, 25(13), 7514-7522.

10 E. Glynos, V. Sboros and V. Koutsos, Mater. Sci. Eng., B, 2009, 165 (3), 231-234.

11 V. Sboros, E. Glynos, S. D. Pye, C. M. Moran, M. Butler, J. A. Ross, W. N. McDicken and V. Koutsos, Ultrasonics, 2007, 46(4), 349-354.

12 P. Dayton, A. Klibanov, G. Brandenburger and K. Ferrara, Ultrasound Med. Biol., 1999, 25(8), 1195-1201.

13 A. L. Klibanov, Adv. Drug Delivery Rev., 1999, 37(1-3), 139-157.

14 E. C. Unger, E. Hersh, M. Vannan, T. O. Matsunaga and T. McCreery, Prog. Cardiovasc. Dis., 2001, 44(1), 45-54.

15 J. R. Lindner, M. P. Coggins, S. Kaul, A. L. Klibanov, G. H. Brandenburger and K. Ley, Circulation, 2000, 101(6), 668-675.

16 T. R. Porter and F. Xie, Prog. Cardiovasc. Dis., 2001, 44(2), 101-110.

17 T. R. Porter and F. Xie, Echocardiography, 2001, 18(4), 349-353.

18 E. C. Unger, E. Hersh, M. Vannan and T. McCreery, Echocardiography, 2001, 18(4), 355-361.

19 M. J. K. Blomley, J. C. Cooke, E. C. Unger, M. J. Monaghan and D. O. Cosgrove, Br. Med. J., 2001, 322(7296), 1222-1225.

20 M. A. Borden and M. L. Longo, Langmuir, 2002, 18(24), 9225-9233.

21 S. Mayer and P. A. Grayburn, Prog. Cardiovasc. Dis., 2001, 44(1), 3344.

22 D. M. Skyba and S. Kaul, Coron. Artery Dis., 2000, 11(3), 211-219.

23 P. Dayton, D. Pearson, J. Clark, S. Simon, P. Schumann, R. Zutshi, T. Matsunaga and K. Ferrara, Mol. Imaging, 2004, 3(2), 25-34.

24 D. Needham and D. H. Kim, Colloids Surf., B, 2000, 18(3-4), 183195.

25 J. E. McKendry, C. A. Grant, B. R. G. Johnson, P. L. Coletta, J. A. Evans and S. D. Evans, Bubble Sci., Eng. Technol., 2010, 2, $48-54$.

26 A. K. Bembey, M. L. Oyen, A. J. Bushby and A. Boyde, Philos. Mag., 2006, 86(33), 5691-5703.

27 S. Moreno-Flores, R. Benitez, M. Vivanco and J. L. Toca-Herrera, Nanotechnology, 2010, 21(44), 445101.

28 D. Tranchida, Z. Kiflie, S. Acierno and S. Piccarolo, Meas. Sci. Technol., 2009, 20(9), 095702.

29 S. K. Kaliappan and B. Cappella, Polymer, 2005, 46(25), 1141611423.

30 S. Garcia-Manyes, G. Oncins and F. Sanz, Biophys. J., 2005, 89(6), $4261-4274$.

31 F. Yarrow, T. J. H. Vlugt, J. P. J. M. van der Eerden and M. M. E. Snel, J. Cryst. Growth, 2005, 275(1-2), e1417-e1421.

32 A. C. Fischer-Cripps, Mater. Sci. Eng., A, 2004, 385(1-2), 74-82.

33 H. Isaksson, S. Nagao, M. MaLkiewicz, P. Julkunen, R. Nowak and J. S. Jurvelin, J. Biomech., 2010, 43(12), 2410-2417.

34 Z. Wu, T. A. Baker, T. C. Ovaert and G. L. Niebur, J. Biomech., 2011, 44(6), 1066-1072.

35 V. Vadillo-Rodriguez, T. J. Beveridge and J. R. Dutcher, J. Bacteriol., 2008, 190(12), 4225-4232.

36 P. C. Y. Lau, J. R. Dutcher, T. J. Beveridge and J. S. Lam, Biophys. J., 2009, 96(7), 2935-2948.

37 R. Goodall and T. W. Clyne, Acta Mater., 2006, 54(20), 5489-5499.

38 R. E. Cohen, C. J. Hooley and N. G. McCrum, J. Biomech., 1976, 9 (4), 175-184.

39 J. L. Hutter and J. Bechhoefer, Rev. Sci. Instrum., 1993, 64(7), 18681873.

40 V. Sboros, E. Glynos, S. D. Pye, C. M. Moran, M. Butler, J. Ross, R. Short, W. N. McDicken and V. Koutsos, Ultrasound Med. Biol., 2006, 32(4), 579-585.

\footnotetext{
\$ The Leeds Microbubble Consortium consists of: Louise Coletta, Pam Jones and Alex Markham (Leeds Institute for Molecular Medicine); Richard Bushby (Chemistry); Neil Thomson (Oral biology); Stephen Freear (Electrical and Electronic Engineering) and Stephen D Evans the school of Physics and Astronomy.
} 